\title{
A 2D WAVE FINITE ELEMENT-BASED SUPERELEMENT FORMULATION FOR ACOUSTIC ANALYSIS OF CAVITIES OF ARBITRARY SHAPES
}

\author{
J.-M. Mencik ${ }^{1}$, D. Duhamel ${ }^{2}$, and M.-L. Gobert ${ }^{1}$ \\ ${ }^{1}$ INSA Centre Val de Loire, Université François Rabelais de Tours, LMR EA 2640 \\ Campus de Blois, 3 Rue de la Chocolaterie, CS 23410, 41034 Blois Cedex, France \\ e-mail: \{jean-mathieu.mencik,marie-laure.gobert\}@insa-cvl.fr \\ ${ }^{2}$ Université Paris-Est, Laboratoire Navier, ENPC/IFSTTAR/CNRS \\ 6 et 8 Avenue Blaise Pascal, Cité Descartes, Champs-sur-Marne, 77455 Marne La Vallée Cedex 2, \\ France \\ e-mail: denis.duhamel@enpc.fr
}

Keywords: Wave Finite Element Method, Acoustic Superelements, Substructuring, Mid-Frequencies.

\begin{abstract}
A substructuring technique is proposed which enables fast computation of the acoustic response of arbitrary-shaped $2 \mathrm{D}$ cavities subject to different kinds of excitations. It combines rectangular superelements which are modeled by means of the wave finite element (WFE) method, and arbitrary-shaped superelements modeled using component mode synthesis (CMS). Within the WFE framework, the so-called receptance matrices of rectangular superelements - which link the pressure vectors to the acoustic force vectors over the boundaries - can be derived in an efficient way in terms of wave modes, without the need of explicitly condensing the internal degrees of freedom of the systems. A model reduction strategy is proposed which aims at expressing the receptance matrices with a few wave modes only. The proposed strategy involves enclosing each rectangular superelement in a finite element (FE) layer with a small width. In this way, smoothed pressure fields are likely to occur over the WFE superelements, hence enabling these superelements to be described with a few wave modes only. By considering those WFE-based rectangular superelements surrounded by FE layers, this yields the so-called hybrid WFE/FE superelements whose dynamic stiffness matrices can be computed in a very fast way. Modeling a whole arbitrary-shaped acoustic cavity follows from conventional assembly procedure between hybrid WFE/FE superelements, CMS superelements and other FE components. Numerical experiments are carried out to highlight the relevance of the proposed substructuring technique.
\end{abstract}




\section{INTRODUCTION}

Developing fast and accurate numerical tools for assessing the acoustic behavior of arbitraryshaped cavities over wide frequency ranges appears to be an open industrial challenge, leading the way of proposing efficient solutions to noise reduction. Popular examples of such cavities are passenger compartments in cars, and aircraft cabins. The finite element (FE) and boundary element (BE) methods are popular techniques for computing internal acoustic problems, but have however to face several severe issues as soon as the number of degrees of freedom (DOFs) involved reaches excessive values. The issue lies in the computation of large-sized matrix systems (FE framework), or the consideration of dense matrices which require high memory storage (BE framework), at many discrete frequencies. This means numerical techniques which are either time consuming or inaccurate.

Reduction methods like FE-based substructuring or wave-based analytical techniques seem to constitute efficient alternatives to the conventional FE and BE methods. Among the FE-based substructuring techniques, one can mention the Craig-Bampton method [1] which focuses on partitioning a whole system into several components and modeling these components by means of static modes and fixed interface modes. The tough issue when dealing with the CB method concerns the determination of the fixed interface modes which would need to be retained for assessing the acoustic behavior of cavities with accurate precision. This explains why, in practice, many fixed interface modes are usually retained to ensure the convergence of the CB solutions, hence impacting the CPU times required for modeling the CB components. On the other hand, Trefftz techniques like the wave based method (WBM) [2] aim a using a few analytical wave functions to describe the acoustic behavior of 2D cavities, even in the short wavelength domain. In this sense, they appear to be efficient in terms of CPU times. Their disadvantage, however, is that they invoke dense matrices in the same way as the BE method.

A new FE-based substructuring technique is proposed here which overcomes the aforementioned issues. It makes use of the wave finite element (WFE) method which aims at assessing numerical wave modes traveling along periodic systems $[3,4,5]$. Within this framework, a so-called hybrid WFE/FE superelement is formulated which consists in a rectangular acoustic domain, with a periodic FE mesh, surrounded by an arbitrary-meshed FE layer with a small width. The interesting feature of this superelement is that the receptance matrix of the rectangular domain - i.e., which links the vector of nodal pressures to that of the nodal forces over the boundary - can be expressed by means of a small number of wave modes. The fact that a few wave modes are only used is explained because the pressure field is expected to be smooth over the rectangular domain, it being understood that local singularities near excitations and boundary conditions are managed by the FE layer. In doing so, the proposed superelement can be modeled using a dynamic stiffness matrix of small size whose computation doesn't require excessive CPU times. Modeling a whole acoustic cavity follows from conventional FE procedure by assembling several hybrid WFE/FE superelements, as well as CMS-based superelements and other FE components together.

The rest of the paper is organized as follows. In Section 2, the basics of the WFE method are recalled which concern the description of wave modes traveling along a rectangular acoustic domain with a periodic FE mesh. Also, the strategy for computing the forced response of periodic systems is presented. In Section 3, the WFE-based procedure for expressing the receptance matrix of a rectangular domain is proposed. Also, the formulation of the WFE/FE hybrid superelement is proposed. Finally, numerical experiments are carried out in Section 4 to highlight the computational efficiency of the proposed approach in comparison with the conventional $\mathrm{CB}$ 
method.

\section{WFE METHOD}

\subsection{Wave mode computation}

The basics of the WFE method, which concern the description of wave modes traveling along a one-dimensional periodic system, are recalled hereafter. Within the present scope, emphasis will be placed on the study of rectangular acoustic domains with a FE mesh which is periodic along one straight direction, i.e., composed of identical substructures connected to each other, as shown in Figure 1. Such a rectangular acoustic domain is supposed to be excited by harmonic excitations (the angular frequency being denoted as $\omega$ ) which are confined to the left and right edges only, and modeled in terms of two vectors of acoustic forces $\mathbf{F}_{0}$ and $\mathbf{F}_{0}^{\star}$ (see Figure 1).

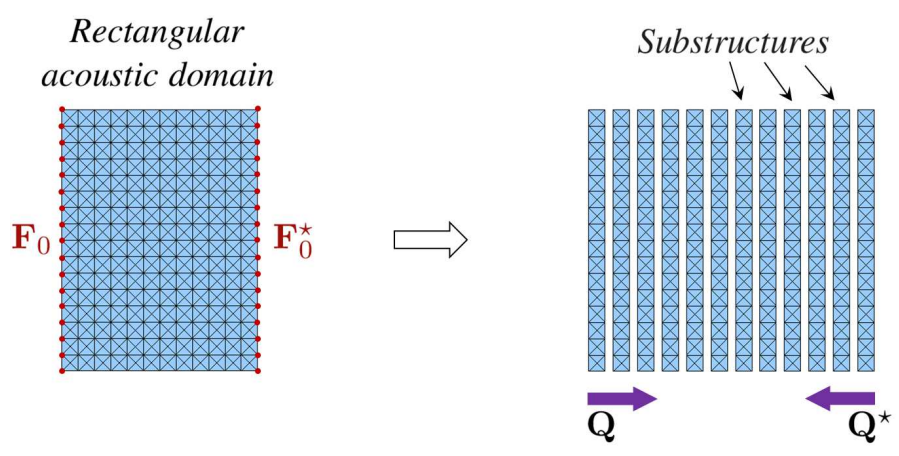

Figure 1: Rectangular acoustic domain with a periodic FE mesh, and related WFE modeling.

For the purpose of wave mode description, the WFE method requires one to know the acoustic mass matrix $\mathbf{M}$ and acoustic stiffness matrix $\mathbf{K}$ of a substructure, and ultimately the condensed dynamic stiffness matrix $\mathbf{D}^{*}=\mathbf{D}_{\mathrm{BB}}-\mathbf{D}_{\mathrm{BI}} \mathbf{D}_{\mathrm{II}}^{-1} \mathbf{D}_{\mathrm{IB}}$, where $\mathbf{D}=-\omega^{2} \mathbf{M}+\mathbf{K}$ is the dynamic stiffness matrix of the substructure Here, the subscript $B$ refers to the degrees of freedom (DOFs) on the left and right edges of the substructure, while the subscript I refers to the internal DOFs (i.e., which do not belong to the left and right edges). Denote as $n$ the number of DOFs on the left (or right) edge of the substructure. Following the WFE framework $[4,3]$, the wave modes are sought as the solutions of an eigenproblem of the form $\mathbf{S} \phi_{j}=\mu_{j} \phi_{j}$ in which $\mathbf{S}$ is a $2 n \times 2 n$ symplectic matrix expressed by

$$
\mathbf{S}=\left[\begin{array}{c|c}
-\mathbf{D}_{\mathrm{LR}}^{*-1} \mathbf{D}_{\mathrm{LL}}^{*} & -\mathbf{D}_{\mathrm{LR}}^{*-1} \\
\hline \mathbf{D}_{\mathrm{RL}}^{*}-\mathbf{D}_{\mathrm{RR}}^{*} \mathbf{D}_{\mathrm{LR}}^{*-1} \mathbf{D}_{\mathrm{LL}}^{*} & -\mathbf{D}_{\mathrm{RR}}^{*} \mathbf{D}_{\mathrm{LR}}^{*-1}
\end{array}\right]
$$

where the subscripts $\mathrm{L}$ and $\mathrm{R}$ refer to the left and right edges of the substructure, respectively. The eigenvectors of $\mathbf{S}$ - namely, $\left\{\boldsymbol{\phi}_{j}\right\}_{j}$ - are the so-called wave shapes which are partitioned into pressure and force components as $\phi_{j}=\left[\left(\phi_{j}\right)_{\mathrm{p}}^{T}\left(\phi_{j}\right)_{\mathrm{F}}^{T}\right]^{T}$. Also, since the matrix $\mathbf{S}$ is symplectic, its eigenvalues come in pairs as $\left(\mu_{j}, 1 / \mu_{j}\right)$.

From the numerical point of view, a so-called $\mathbf{S}+\mathbf{S}^{-1}$ transformation of the eigenproblem (1) can be considered $[6,7]$ for accurately computing the eigensolutions $\left\{\mu_{j}\right\}_{j}$ and $\left\{\phi_{j}\right\}_{j}$. The interesting feature of the $\mathbf{S}+\mathbf{S}^{-1}$ transformation lies in the use of skew-symmetric matrices $\left(\mathbf{N}^{\prime} \mathbf{J} \mathbf{L}^{\prime T}+\mathbf{L}^{\prime} \mathbf{J} \mathbf{N}^{\prime} T\right)$ and $\mathbf{L}^{\prime} \mathbf{J L}{ }^{\prime} T$, leading to the following eigenproblem whose eigenvalues are of the form $\lambda_{j}=\mu_{j}+1 / \mu_{j}$ :

$$
\left(\left(\mathbf{N}^{\prime} \mathbf{J L}^{\prime T}+\mathbf{L}^{\prime} \mathbf{J} \mathbf{N}^{\prime} T\right)-\lambda_{j} \mathbf{L}^{\prime} \mathbf{J} \mathbf{L}^{\prime}\right) \mathbf{z}_{j}=\mathbf{0}
$$


where

$$
\mathbf{L}^{\prime}=\left[\begin{array}{cc}
\mathbf{0} & \mathbf{I}_{n} \\
\mathbf{D}_{\mathrm{LR}}^{*} & \mathbf{0}
\end{array}\right] \quad, \quad \mathbf{N}^{\prime}=\left[\begin{array}{cc}
\mathbf{D}_{\mathrm{RL}}^{*} & \mathbf{0} \\
-\left(\mathbf{D}_{\mathrm{LL}}^{*}+\mathbf{D}_{\mathrm{RR}}^{*}\right) & -\mathbf{I}_{n}
\end{array}\right] \quad, \quad \mathbf{J}=\left[\begin{array}{cc}
\mathbf{0} & \mathbf{I}_{n} \\
-\mathbf{I}_{n} & \mathbf{0}
\end{array}\right] .
$$

Once the eigenvalues $\left\{\lambda_{j}\right\}_{j}$ in Eq. (2) are found, the original eigenvalues $\left\{\mu_{j}\right\}_{j}$ are found analytically by solving a quadratic equation of the form

$$
x^{2}-\lambda_{j} x+1=0 .
$$

Also, once the eigenvectors $\left\{\mathbf{z}_{j}\right\}_{j}$ in Eq. (2) are found, the original eigenvectors $\left\{\boldsymbol{\phi}_{j}\right\}_{j}$ are found analytically as follows [7]:

$$
\phi_{j}=\left[\begin{array}{cc}
\mathbf{I}_{n} & \mathbf{0} \\
\mathbf{D}_{\mathrm{RR}}^{*} & \mathbf{I}_{n}
\end{array}\right] \mathbf{w}_{j}^{\prime} \quad \text { where } \quad \mathbf{w}_{j}^{\prime}=\mathbf{J}\left(\mathbf{L}^{\prime T}-\frac{1}{\mu_{j}} \mathbf{N}^{\prime T}\right) \mathbf{z}_{j} .
$$

\subsection{Conventions and matrix notations}

As was mentioned in Section 2.1, the eigenvalues of the matrix $\mathbf{S}$ are pairs $\left(\mu_{j}, 1 / \mu_{j}\right)$. This leads to the consideration of $n$ right-going wave modes for which $\left|\mu_{j}\right| \leq 1$, and $n$ left-going wave modes for which $\left|\mu_{j}\right| \geq 1$. Regarding the case $\left|\mu_{j}\right|=1$, the right-going (resp. left-going) wave modes are defined so that $(i \omega / 4) \phi_{j}^{H} \mathbf{J} \phi_{j}>1$ (resp. $(i \omega / 4) \phi_{j}^{H} \mathbf{J} \phi_{j}<1$ ), which means that the related energy flows [8] are conveyed towards the right (resp. left) direction of the system.

For the sake of clarity, the sets of right- and left- going wave modes will be denoted as $\left\{\left(\mu_{j}, \phi_{j}\right)\right\}_{j=1, \ldots, n}$ and $\left\{\left(\mu_{j}^{\star}, \phi_{j}^{\star}\right)\right\}_{j=1, \ldots, n}$, where it is understood that $\mu_{j}^{\star}=1 / \mu_{j} \forall j \in\{1, \ldots, n\}$. Also, by considering Eq. (5), the wave shapes $\phi_{j}$ and $\phi_{j}^{\star}$ are expressed as

$$
\phi_{j}=\left[\begin{array}{cc}
\mathbf{I}_{n} & \mathbf{0} \\
\mathbf{D}_{\mathrm{RR}}^{*} & \mathbf{I}_{n}
\end{array}\right] \mathbf{J}\left(\mathbf{L}^{\prime T}-\mu_{j}^{\star} \mathbf{N}^{\prime T}\right) \mathbf{z}_{j} \quad, \quad \boldsymbol{\phi}_{j}^{\star}=\left[\begin{array}{cc}
\mathbf{I}_{n} & \mathbf{0} \\
\mathbf{D}_{\mathrm{RR}}^{*} & \mathbf{I}_{n}
\end{array}\right] \mathbf{J}\left(\mathbf{L}^{\prime T}-\mu_{j} \mathbf{N}^{\prime T}\right) \mathbf{z}_{j},
$$

where $\mathbf{z}_{j}$ is an eigenvector of Eq. (2) corresponding to the eigenvalue $\lambda_{j}$. In matrix form, the wave modes can thus be written as

$$
\boldsymbol{\mu}=\operatorname{diag}\left\{\mu_{j}\right\}_{j=1, \ldots, n} \quad, \quad \boldsymbol{\mu}^{\star}=\boldsymbol{\mu}^{-1} \quad, \quad \boldsymbol{\Phi}=\left[\phi_{1} \ldots \phi_{n}\right] \quad, \quad \boldsymbol{\Phi}^{\star}=\left[\boldsymbol{\phi}_{1}^{\star} \ldots \boldsymbol{\phi}_{n}^{\star}\right],
$$

with the property that $\|\boldsymbol{\mu}\|_{2} \leq 1\left(\|.\|_{2}\right.$ being the $2-$ norm $)$. Also, the matrices $\boldsymbol{\Phi}$ and $\boldsymbol{\Phi}^{\star}$ are to be partitioned into pressure and force components, as follows:

$$
\boldsymbol{\Phi}=\left[\begin{array}{c}
\boldsymbol{\Phi}_{\mathrm{p}} \\
\boldsymbol{\Phi}_{\mathrm{F}}
\end{array}\right] \quad, \quad \boldsymbol{\Phi}^{\star}=\left[\begin{array}{c}
\boldsymbol{\Phi}_{\mathrm{p}}^{\star} \\
\boldsymbol{\Phi}_{\mathrm{F}}^{\star}
\end{array}\right],
$$

where $\Phi_{\mathrm{p}}, \Phi_{\mathrm{F}}, \Phi_{\mathrm{p}}^{\star}$ and $\Phi_{\mathrm{F}}^{\star}$ are $n \times n$ matrices which are full rank [9]. 


\subsection{Forced response computation}

Within the framework of the WFE method, the vectors of pressures and acoustic forces of an assembly of $N$ substructures like the one displayed in Figure 1 are expressed in terms of wave shapes, as follows [10]:

$$
\mathbf{p}^{(k)}=\boldsymbol{\Phi}_{\mathrm{p}} \boldsymbol{\mu}^{k-1} \mathbf{Q}+\boldsymbol{\Phi}_{\mathrm{p}}^{\star} \boldsymbol{\mu}^{N-k+1} \mathbf{Q}^{\star} \quad, \quad \pm \mathbf{F}^{(k)}=\boldsymbol{\Phi}_{\mathrm{F}} \boldsymbol{\mu}^{k-1} \mathbf{Q}+\boldsymbol{\Phi}_{\mathrm{F}}^{\star} \boldsymbol{\mu}^{N-k+1} \mathbf{Q}^{\star} \quad k=1, \ldots, N+1 .
$$

where the subscript $(k)$ designates any substructure boundary, i.e., either a coupling interface/edge between two consecutive substructures $k-1$ and $k$, or the left/right edge of the whole domain; also, $\mathrm{Q}$ and $\mathrm{Q}^{\star}$ are vectors of wave amplitudes which are respectively defined at the left and right edges of the whole domain, i.e., at the substructure boundaries $(1)$ and $(N+1)$. In Eq. (9), the sign ahead of $\mathbf{F}^{(k)}$ should be negative in case when a left boundary is considered, and positive for a right boundary.

The problem behind the computation of the forced response of the acoustic domain can be stated as to find the vectors of wave amplitudes $\mathrm{Q}$ and $\mathrm{Q}^{\star}$. This is achieved by considering the boundary conditions (BCs) on the left and right edges, the other substructure boundaries being assumed to be free from excitations sources (see Figure 1). Those BCs may involve prescribed pressures or acoustic forces. In view of the subsequent developments made in this paper, we will restrict the present analysis to the study of two vectors of prescribed forces $\mathbf{F}_{0}$ and $\mathbf{F}_{0}^{\star}$ that apply over the substructure boundaries $(1)$ and $(N+1)$, respectively, as shown in Figure 1 . The related wave expansion, Eq. (9), yields

$$
-\mathbf{F}_{0}=\boldsymbol{\Phi}_{\mathrm{F}} \mathrm{Q}+\boldsymbol{\Phi}_{\mathrm{F}}^{\star} \boldsymbol{\mu}^{N} \mathbf{Q}^{\star} \quad, \quad \mathbf{F}_{0}^{\star}=\boldsymbol{\Phi}_{\mathrm{F}} \mathbf{Q} \boldsymbol{\mu}^{N}+\boldsymbol{\Phi}_{\mathrm{F}}^{\star} \mathbf{Q}^{\star} .
$$

As a result, a wave-based matrix system can be derived whose resolution provides the vectors of wave amplitudes $\mathbf{Q}$ and $\mathbf{Q}^{\star}$. According to Eq. (10), it is expressed as follows:

$$
\mathbf{A}\left[\begin{array}{c}
\mathbf{Q} \\
\mathbf{Q}^{\star}
\end{array}\right]=\mathbf{B}\left[\begin{array}{c}
\mathbf{F}_{0} \\
\mathbf{F}_{0}^{\star}
\end{array}\right]
$$

where $\mathbf{A}$ and $\mathbf{B}$ are $2 n \times 2 n$ matrices defined as

$$
\mathbf{A}=\left[\begin{array}{cc}
\mathbf{I} & \boldsymbol{\Phi}_{\mathrm{F}}^{-1} \boldsymbol{\Phi}_{\mathrm{F}}^{\star} \boldsymbol{\mu}^{N} \\
\boldsymbol{\Phi}_{\mathrm{F}}^{\star-1} \boldsymbol{\Phi}_{\mathrm{F}} \boldsymbol{\mu}^{N} & \mathbf{I}
\end{array}\right] \quad, \quad \mathbf{B}=\left[\begin{array}{cc}
-\boldsymbol{\Phi}_{\mathrm{F}}^{-1} & \mathbf{0} \\
\mathbf{0} & \boldsymbol{\Phi}_{\mathrm{F}}^{\star-1}
\end{array}\right] .
$$

To derive the matrices $\mathbf{A}$ and $\mathbf{B}$, it has been assumed that the matrices $\boldsymbol{\Phi}_{\mathrm{F}}$ and $\boldsymbol{\Phi}_{\mathrm{F}}^{\star}$ are invertible (a proof of this statement is brought in ref. [9]). The motivation behind the use of the matrix inverses $\Phi_{\mathrm{F}}^{-1}$ and $\Phi_{\mathrm{F}}^{\star-1}$ is to make the matrix $\mathbf{A}$ well-conditioned [9]. The determination of the vectors of wave amplitudes $\mathrm{Q}$ and $\mathrm{Q}^{\star}$ hence follows from Eq. (11):

$$
\left[\begin{array}{c}
\mathbf{Q} \\
\mathbf{Q}^{\star}
\end{array}\right]=\mathbf{A}^{-1} \mathbf{B}\left[\begin{array}{c}
\mathbf{F}_{0} \\
\mathbf{F}_{0}^{\star}
\end{array}\right]
$$

\section{SUPERELEMENT MODELING}

\subsection{Introduction}

Consider now a rectangular acoustic domain which undergoes acoustic forces over its left/right and bottom/top edges, as depicted in Figure 2. The motivation behind the proposed study is to 
express a so-called receptance matrix $\mathbf{H}$ which links the vector of pressures of the boundary nodes $\mathrm{p}_{\mathrm{B}}$ to that of the vector of acoustic forces $\mathbf{F}_{\mathrm{B}}$, where the subscript $\mathrm{B}$ refers to the nodes belonging to the left/right and bottom/top edges. This relation is expressed by $\mathbf{p}_{\mathrm{B}}=\mathbf{H F}_{\mathrm{B}}$. The key idea here is to express the receptance matrix $\mathbf{H}$ in terms of wave modes (Section 2) traveling along both horizontal and vertical directions, as shown in Figure 2. This yields a superelement modeling whose derivation follows from the well-known superposition principle (see Section 3.2).

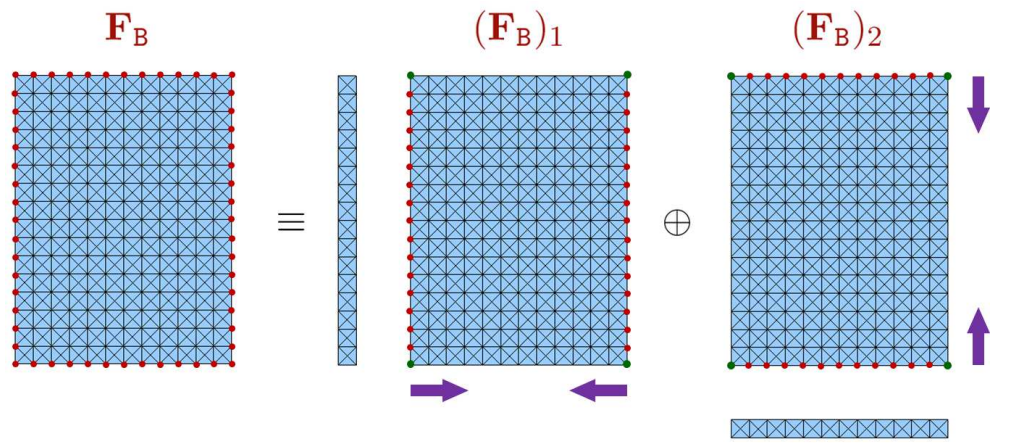

Figure 2: Illustration of the superposition principle involving a vector of acoustic force decomposed into two ones acting respectively along the left/right and bottom/top edges.

\subsection{Receptance matrix}

The key idea behind the present approach is to decompose the vector of acoustic forces $\mathbf{F}_{\mathrm{B}}$ into two states of excitation $\left(\mathbf{F}_{\mathrm{B}}\right)_{1}$ and $\left(\mathbf{F}_{\mathrm{B}}\right)_{2}$ that operate, respectively, on the vertical and horizontal edges (see Figure 2). This means two WFE modelings (namely, 1 and 2), and related substructures, for assessing the wave propagation along the horizontal and vertical directions. For each WFE modeling $i(i=1,2)$, the number of right/left- (or top/bottom-) going wave modes involved is $n_{i}-n_{1}$ and $n_{2}$ being the number of DOFs contained over the left/right edge and bottom/top edge, respectively - while the number of substructures is $N_{i}-N_{1}$ and $N_{2}$ being the number of substructures involved along the horizontal and vertical directions, respectively. For the sake of simplicity, it is assumed that the boundary nodes of the "vertical" substructures (WFE modeling 1) are only confined to their left and right edges, while those of the "horizontal" substructures (WFE modeling 2) are only confined to their bottom and top edges (see Figure 2). By considering the superposition principle, this yields

$$
\mathbf{F}_{\mathrm{B}}=\left(\mathbf{F}_{\mathrm{B}}\right)_{1}+\left(\mathbf{F}_{\mathrm{B}}\right)_{2} \quad \Rightarrow \quad \mathbf{p}_{\mathrm{B}}=\left(\mathbf{p}_{\mathrm{B}}\right)_{1}+\left(\mathbf{p}_{\mathrm{B}}\right)_{2}
$$

This means that the vector of pressures $\mathrm{p}_{\mathrm{B}}$ produced by the vector of acoustic forces $\mathbf{F}_{\mathrm{B}}$ is expressed as the sum of two vectors $\left(\mathbf{p}_{\mathrm{B}}\right)_{1}$ and $\left(\mathbf{p}_{\mathrm{B}}\right)_{2}$ which are respectively induced by the vectors of acoustic forces $\left(\mathbf{F}_{\mathrm{B}}\right)_{1}$ and $\left(\mathbf{F}_{\mathrm{B}}\right)_{2}$ as acting independently on the acoustic domain. It should be noticed that the vectors $\mathbf{F}_{\mathrm{B}},\left(\mathbf{F}_{\mathrm{B}}\right)_{1},\left(\mathbf{F}_{\mathrm{B}}\right)_{2}, \mathbf{p}_{\mathrm{B}},\left(\mathbf{p}_{\mathrm{B}}\right)_{1}$ and $\left(\mathbf{p}_{\mathrm{B}}\right)_{2}$ have the same size, i.e., $n_{\mathrm{B}} \times 1$, where $n_{\mathrm{B}}=2\left(n_{1}-1\right)+2\left(n_{2}-1\right)$ represents the number of boundary DOFs of the rectangular domain. Also, it should be pointed out that the vectors $\left(\mathbf{F}_{\mathrm{B}}\right)_{1}$ and $\left(\mathbf{F}_{\mathrm{B}}\right)_{2}$ contain, respectively, $2\left(n_{2}-1\right)$ and $2\left(n_{1}-1\right)$ zero-components. Those vectors are expressed as

$$
\left(\mathbf{F}_{\mathrm{B}}\right)_{1}=\mathcal{P}_{1} \mathbf{F}_{\mathrm{B}} \quad, \quad\left(\mathbf{F}_{\mathrm{B}}\right)_{2}=\mathcal{P}_{2} \mathbf{F}_{\mathrm{B}}
$$


where $\mathcal{P}_{1}$ and $\mathcal{P}_{2}$ are two $n_{\mathrm{B}} \times n_{\mathrm{B}}$ matrices which relate the partitioning depicted in Figure 2 and which are defined so that

$$
\mathcal{P}_{1}+\mathcal{P}_{2}=\mathbf{I}_{n_{\mathrm{B}}}
$$

In fact, the matrices $\mathcal{P}_{1}$ and $\mathcal{P}_{2}$ are diagonal and mostly composed of 1 and 0 components to enforce the fact that certain nodes are excited, while other are free. Other components equal to $1 / 2$ should also be considered which specifically address the acoustic forces at the corners of the domain (Figure 2), it being understood they are to be equally partitioned between the states of excitation $\left(\mathbf{F}_{\mathrm{B}}\right)_{1}$ and $\left(\mathbf{F}_{\mathrm{B}}\right)_{2}$.

The acoustic behavior of the rectangular domain, as subjected either to $\left(\mathbf{F}_{\mathrm{B}}\right)_{1}$ or $\left(\mathbf{F}_{\mathrm{B}}\right)_{2}$, can be assessed using the strategy depicted in Section 2.3. For each state $i$, the strategy consists in considering $n_{i} \times 1$ vectors of acoustic forces $\left(\mathbf{F}_{0}\right)_{i}$ and $\left(\mathbf{F}_{0}^{\star}\right)_{i}$ acting over the left/right or bottom/top edges. These vectors are linked to the full vector of acoustic forces $\left(\mathbf{F}_{\mathrm{B}}\right)_{i}$ as follows:

$$
\left[\begin{array}{c}
\left(\mathbf{F}_{0}\right)_{i} \\
\left(\mathbf{F}_{0}^{\star}\right)_{i}
\end{array}\right]=\left(\mathcal{B}_{\mathrm{F}}\right)_{i}\left(\mathbf{F}_{\mathrm{B}}\right)_{i} \quad i=1,2,
$$

where $\left(\mathcal{B}_{\mathrm{F}}\right)_{i}$ is a $2 n_{i} \times n_{\mathrm{B}}$ Boolean matrix. According to Eqs. (13), (15) and (17), the vectors of wave amplitudes are expressed as

$$
\left[\begin{array}{c}
\mathbf{Q}_{i} \\
\mathbf{Q}_{i}^{\star}
\end{array}\right]=\mathbf{A}_{i}^{-1} \mathbf{B}_{i}\left(\mathcal{B}_{\mathrm{F}}\right)_{i} \mathcal{P}_{i} \mathbf{F}_{\mathrm{B}} \quad i=1,2,
$$

where

$$
\mathbf{A}_{i}=\left[\begin{array}{cc}
\mathbf{I}_{n_{i}} & \left(\boldsymbol{\Phi}_{\mathrm{F}}\right)_{i}^{-1}\left(\boldsymbol{\Phi}_{\mathrm{F}}^{\star}\right)_{i} \boldsymbol{\mu}_{i}^{N_{i}} \\
\left(\boldsymbol{\Phi}_{\mathrm{F}}^{\star}\right)_{i}^{-1}\left(\boldsymbol{\Phi}_{\mathrm{F}}\right)_{i} \boldsymbol{\mu}_{i}^{N_{i}} & \mathbf{I}_{n_{i}}
\end{array}\right] \quad, \quad \mathbf{B}_{i}=\left[\begin{array}{cc}
-\left(\boldsymbol{\Phi}_{\mathrm{F}}\right)_{i}^{-1} & \mathbf{0}_{n_{i} \times n_{i}} \\
\mathbf{0}_{n_{i} \times n_{i}} & \left(\boldsymbol{\Phi}_{\mathrm{F}}^{\star}\right)_{i}^{-1}
\end{array}\right] \quad i=1,2 .
$$

In a similar way as Eq. (17), there exists a relation which links the vector of nodal pressures $\mathbf{p}_{i}^{\left(k_{i}\right)}$ on a given substructure boundary $\left(k_{i}\right)\left(k_{i}=1, \ldots, N_{i}+1\right)$ with the vector of nodal pressures $\left(\mathbf{p}_{\mathrm{B}}\right)_{i}$ on the edges of the rectangular domain. This yields

$$
\left[\begin{array}{c}
\mathbf{p}_{i}^{(1)} \\
\mathcal{L}_{i}^{(2)} \mathbf{p}_{i}^{(2)} \\
\vdots \\
\mathcal{L}_{i}^{\left(N_{i}\right)} \mathbf{p}_{i}^{\left(N_{i}\right)} \\
\mathbf{p}_{i}^{\left(N_{i}+1\right)}
\end{array}\right]=\left(\mathcal{B}_{\mathbf{p}}\right)_{i}\left(\mathbf{p}_{\mathrm{B}}\right)_{i} \quad i=1,2,
$$

where $\mathcal{L}^{\left(k_{i}\right)}\left(k_{i}=2, \ldots, N_{i}\right)$ is a $2 \times n_{i}$ Boolean matrix which localizes among the DOFs of the substructure boundary $\left(k_{i}\right)$, those belonging to the edges of the domain. The way the matrix $\mathcal{L}^{\left(k_{i}\right)}$ works is highlighted in Figure 3. Notice that both $\mathbf{p}_{i}^{(1)}$ and $\mathbf{p}_{i}^{\left(N_{i}+1\right)}$ are considered in the left-hand side of Eq. (20) since all their components relate nodal pressures on the edges of the domain (see Figure 3). Finally, in Eq. $(20),\left(\mathcal{B}_{\mathrm{p}}\right)_{i}$ is a $n_{\mathrm{B}} \times n_{\mathrm{B}}$ orthogonal Boolean matrix whose purpose is to renumber the rows and columns of the matrix occurring in the left-hand side so as to match the node numbering scheme of the vectors $p_{B}$ and $F_{B}$, Eq. (14), which may be arbitrary a priori.

Invoking the wave expansion (9) into Eq. (20) while using the fact that the matrix $\left(\mathcal{B}_{\mathrm{p}}\right)_{i}$ is orthogonal - i.e., $\left(\mathcal{B}_{\mathrm{p}}\right)_{i}^{-1}=\left(\mathcal{B}_{\mathrm{p}}\right)_{i}^{T}-$, this yields

$$
\left(\mathbf{p}_{\mathrm{B}}\right)_{i}=\left(\mathcal{B}_{\mathrm{p}}\right)_{i}^{T}\left(\mathbf{\Psi}_{\mathrm{p}}\right)_{i}\left[\begin{array}{l}
\mathbf{Q}_{i} \\
\mathbf{Q}_{i}^{\star}
\end{array}\right] \quad i=1,2,
$$




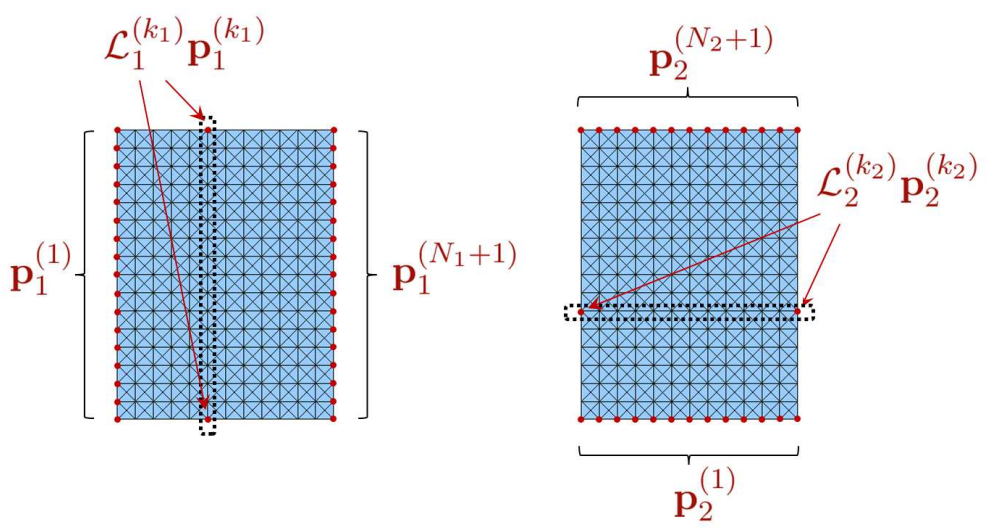

Figure 3: Illustration of the vectors $\mathcal{L}_{1}^{\left(k_{1}\right)} \mathbf{p}_{1}^{\left(k_{1}\right)}$ and $\mathcal{L}_{2}^{\left(k_{2}\right)} \mathbf{p}_{2}^{\left(k_{2}\right)}$.

where $\left(\Psi_{\mathrm{p}}\right)_{i}$ is a $n_{\mathrm{B}} \times 2 n_{i}$ matrix defined by

$$
\left(\boldsymbol{\Psi}_{\mathrm{p}}\right)_{i}=\left[\begin{array}{c|c}
\left(\boldsymbol{\Phi}_{\mathrm{p}}\right)_{i} & \left(\boldsymbol{\Phi}_{\mathrm{p}}^{\star}\right)_{i} \boldsymbol{\mu}_{i}^{N_{i}} \\
\mathcal{L}_{i}^{(2)}\left(\boldsymbol{\Phi}_{\mathrm{p}}\right)_{i} \boldsymbol{\mu}_{i} & \mathcal{L}_{i}^{(2)}\left(\boldsymbol{\Phi}_{\mathrm{p}}^{\star}\right)_{i} \boldsymbol{\mu}_{i}^{N_{i}-1} \\
\vdots & \vdots \\
\mathcal{L}_{i}^{\left(N_{i}\right)}\left(\boldsymbol{\Phi}_{\mathrm{p}}\right)_{i} \boldsymbol{\mu}_{i}^{N_{i}-1} & \mathcal{L}_{i}^{\left(N_{i}\right)}\left(\boldsymbol{\Phi}_{\mathrm{p}}^{\star}\right)_{i} \boldsymbol{\mu}_{i} \\
\left(\boldsymbol{\Phi}_{\mathrm{p}}\right)_{i} \boldsymbol{\mu}_{i}^{N_{i}} & \left(\boldsymbol{\Phi}_{\mathrm{p}}^{\star}\right)_{i}
\end{array}\right] \quad i=1,2
$$

By considering Eqs. (21) and (18), a relation linking the vector of pressures $\left(\mathbf{p}_{\mathrm{B}}\right)_{i}$ and acoustic forces $\mathbf{F}_{\mathrm{B}}$ can be derived as follows:

$$
\left(\mathbf{p}_{\mathrm{B}}\right)_{i}=\left(\mathcal{B}_{\mathrm{p}}\right)_{i}^{T}\left(\boldsymbol{\Psi}_{\mathrm{p}}\right)_{i} \mathbf{A}_{i}^{-1} \mathbf{B}_{i}\left(\mathcal{B}_{\mathrm{F}}\right)_{i} \mathcal{P}_{i} \mathbf{F}_{\mathrm{B}} \quad i=1,2 .
$$

The derivation of the receptance matrix $\mathbf{H}-$ defined as $\mathbf{p}_{\mathrm{B}}=\mathbf{H F}_{\mathrm{B}}$ where $\mathbf{p}_{\mathrm{B}}=\left(\mathbf{p}_{\mathrm{B}}\right)_{1}+\left(\mathbf{p}_{\mathrm{B}}\right)_{2}$ - finally follows as

$$
\mathbf{H}=\left(\mathcal{B}_{\mathrm{p}}\right)_{1}^{T}\left(\boldsymbol{\Psi}_{\mathrm{p}}\right)_{1} \mathbf{A}_{1}^{-1} \mathbf{B}_{1}\left(\mathcal{B}_{\mathrm{F}}\right)_{1} \mathcal{P}_{1}+\left(\mathcal{B}_{\mathrm{p}}\right)_{2}^{T}\left(\boldsymbol{\Psi}_{\mathrm{p}}\right)_{2} \mathbf{A}_{2}^{-1} \mathbf{B}_{2}\left(\mathcal{B}_{\mathrm{F}}\right)_{2} \mathcal{P}_{2}
$$

\subsection{Model reduction}

\subsubsection{Introduction}

One way to speed up the computation of the receptance matrix $\mathbf{H}$ is to make use of reduced wave mode bases. In this framework, the vectors of pressures and acoustic forces are assessed by means of reduced sets of wave shapes $\left\{\left(\widetilde{\phi}_{\mathrm{p} j}\right)_{i}\right\}_{j=1, \ldots, m_{i}} \cup\left\{\left(\widetilde{\phi}_{\mathrm{p} j}^{\star}\right)_{i}\right\}_{j=1, \ldots, m_{i}}$ and $\left\{\left(\widetilde{\phi}_{\mathrm{F} j}\right)_{i}\right\}_{j=1, \ldots, m_{i}} \cup\left\{\left(\widetilde{\phi}_{\mathrm{F} j}^{\star}\right)_{i}\right\}_{j=1, \ldots, m_{i}}$ extracted from the full sets $\left\{\left(\boldsymbol{\phi}_{\mathrm{p} j}\right)_{i}\right\}_{j=1, \ldots, n_{i}} \cup\left\{\left(\boldsymbol{\phi}_{\mathrm{p} j}^{\star}\right)_{i}\right\}_{j=1, \ldots, n_{i}}$ and $\left\{\left(\phi_{\mathrm{F} j}\right)_{i}\right\}_{j=1, \ldots, n_{i}} \cup\left\{\left(\phi_{\mathrm{F} j}^{\star}\right)_{i}\right\}_{j=1, \ldots, n_{i}}$, where $m_{i} \leq n_{i}$. This yields a small sized $m_{i} \times m_{i}$ ma$\operatorname{trix} \widetilde{\boldsymbol{\mu}}_{i}=\operatorname{diag}\left\{\left(\widetilde{\mu}_{j}\right)_{i}\right\}_{j=1, \ldots, m_{i}}$, and small sized $n_{i} \times m_{i}$ matrices $\left(\widetilde{\boldsymbol{\Phi}}_{\mathrm{p}}\right)_{i}=\left[\left(\widetilde{\boldsymbol{\phi}}_{\mathrm{p} 1}\right)_{i} \ldots\left(\widetilde{\boldsymbol{\phi}}_{\mathrm{p} m_{i}}\right)_{i}\right]$, $\left(\widetilde{\boldsymbol{\Phi}}_{\mathrm{p}}^{\star}\right)_{i}=\left[\left(\widetilde{\boldsymbol{\phi}}_{\mathrm{p} 1}^{\star}\right)_{i} \ldots\left(\widetilde{\boldsymbol{\phi}}_{\mathrm{p} m_{i}}^{\star}\right)_{i}\right],\left(\widetilde{\boldsymbol{\Phi}}_{\mathrm{F}}\right)_{i}=\left[\left(\widetilde{\boldsymbol{\phi}}_{\mathrm{F} 1}\right)_{i} \ldots\left(\widetilde{\boldsymbol{\phi}}_{\mathrm{F} m_{i}}\right)_{i}\right]$ and $\left(\widetilde{\boldsymbol{\Phi}}_{\mathrm{F}}^{\star}\right)_{i}=\left[\left(\widetilde{\boldsymbol{\phi}}_{\mathrm{F} 1}^{\star}\right)_{i} \ldots\left(\widetilde{\boldsymbol{\phi}}_{\mathrm{F} m_{i}}^{\star}\right)_{i}\right]$. As a result, the receptance matrix can be approximated as $\mathbf{H} \approx \widetilde{\mathbf{H}}$, where (cf. Eq. (24)):

$$
\widetilde{\mathbf{H}}=\left(\mathcal{B}_{\mathrm{p}}\right)_{1}^{T}\left(\widetilde{\boldsymbol{\Psi}}_{\mathrm{p}}\right)_{1} \widetilde{\mathbf{A}}_{1}^{-1} \widetilde{\mathbf{B}}_{1}\left(\mathcal{B}_{\mathrm{F}}\right)_{1} \mathcal{P}_{1}+\left(\mathcal{B}_{\mathrm{p}}\right)_{2}^{T}\left(\widetilde{\boldsymbol{\Psi}}_{\mathrm{p}}\right)_{2} \widetilde{\mathbf{A}}_{2}^{-1} \widetilde{\mathbf{B}}_{2}\left(\mathcal{B}_{\mathrm{F}}\right)_{2} \mathcal{P}_{2}
$$


Here, the matrices $\widetilde{\mathbf{A}}_{i}$ and $\widetilde{\mathbf{B}}_{i}$ are to be expressed as follows (cf. Eq. (19)):

$$
\widetilde{\mathbf{A}}_{i}=\left[\begin{array}{cc}
\mathbf{I}_{m_{i}} & \left(\widetilde{\boldsymbol{\Phi}}_{\mathrm{F}}\right)_{i}^{+}\left(\widetilde{\boldsymbol{\Phi}}_{\mathrm{F}}^{\star}\right)_{i} \widetilde{\boldsymbol{\mu}}_{i}^{N_{i}} \\
\left(\widetilde{\boldsymbol{\Phi}}_{\mathrm{F}}^{\star}\right)_{i}^{+}\left(\widetilde{\boldsymbol{\Phi}}_{\mathrm{F}}\right)_{i} \widetilde{\boldsymbol{\mu}}_{i}^{N_{i}} & \mathbf{I}_{m_{i}}
\end{array}\right] \quad, \quad \widetilde{\mathbf{B}}_{i}=\left[\begin{array}{cc}
-\left(\widetilde{\boldsymbol{\Phi}}_{\mathrm{F}}\right)_{i}^{+} & \mathbf{0}_{m_{i} \times n_{i}} \\
\mathbf{0}_{m_{i} \times n_{i}} & \left(\widetilde{\boldsymbol{\Phi}}_{\mathrm{F}}^{\star}\right)_{i}^{+}
\end{array}\right] \quad i=1,2,
$$

where $\left(\widetilde{\boldsymbol{\Phi}}_{\mathrm{F}}\right)_{i}^{+}$and $\left(\widetilde{\boldsymbol{\Phi}}_{\mathrm{F}}^{\star}\right)_{i}^{+}$are the left pseudo-inverse of the matrices $\left(\widetilde{\boldsymbol{\Phi}}_{\mathrm{F}}\right)_{i}$ and $\left(\widetilde{\boldsymbol{\Phi}}_{\mathrm{F}}^{\star}\right)_{i}$. Also, in Eq. (25), the matrices $\left(\widetilde{\Psi}_{\mathrm{p}}\right)_{1}$ and $\left(\widetilde{\Psi}_{\mathrm{p}}\right)_{2}$ are expressed by (cf. Eq. (22)):

$$
\left(\widetilde{\boldsymbol{\Psi}}_{\mathrm{p}}\right)_{i}=\left[\begin{array}{c|c}
\mathcal{L}_{i}^{(1)}\left(\widetilde{\boldsymbol{\Phi}}_{\mathrm{p}}\right)_{i} & \mathcal{L}_{i}^{(1)}\left(\widetilde{\boldsymbol{\Phi}}_{\mathrm{p}}^{\star}\right)_{i} \widetilde{\boldsymbol{\mu}}_{i}^{N_{i}} \\
\mathcal{L}_{i}^{(2)}\left(\widetilde{\boldsymbol{\Phi}}_{\mathrm{p}}\right)_{i} \widetilde{\boldsymbol{\mu}}_{i} & \mathcal{L}_{i}^{(2)}\left(\widetilde{\boldsymbol{\Phi}}_{\mathrm{p}}^{\star}\right)_{i} \widetilde{\boldsymbol{\mu}}_{i}^{N_{i}-1} \\
\vdots & \vdots \\
\mathcal{L}_{i}^{\left(N_{i}+1\right)}\left(\widetilde{\boldsymbol{\Phi}}_{\mathrm{p}}\right)_{i} \widetilde{\boldsymbol{\mu}}_{i}^{N_{i}} & \mathcal{L}_{i}^{\left(N_{i}+1\right)}\left(\widetilde{\boldsymbol{\Phi}}_{\mathrm{p}}^{\star}\right)_{i}
\end{array}\right] \quad i=1,2 .
$$

\subsubsection{Hybrid WFE/FE superelement modeling}

Although interesting, the use of reduced sets of wave shapes $\left\{\left(\widetilde{\phi}_{\mathrm{p} j}\right)_{i}\right\}_{j=1, \ldots, m_{i}} \cup\left\{\left(\widetilde{\phi}_{\mathrm{p} j}^{\star}\right)_{i}\right\}_{j=1, \ldots, m_{i}}$ and $\left\{\left(\widetilde{\phi}_{\mathrm{F} j}\right)_{i}\right\}_{j=1, \ldots, m_{i}} \cup\left\{\left(\widetilde{\phi}_{\mathrm{F} j}^{\star}\right)_{i}\right\}_{j=1, \ldots, m_{i}}$ may be subject to strong issues, e.g., in case when excitation sources are applied on the edges of the rectangular superelements. In this case, the pressure field is likely to be strongly heterogeneous in the vicinity of the excitation sources, meaning that almost the full sets of wave shapes are required to describe the acoustic behavior of the system with accurate precision. To solve this issue, it is proposed to surround any WFEbased rectangular superelement by means of a thin FE layer whose thickness is not necessarily uniform, as shown in Figure 4.

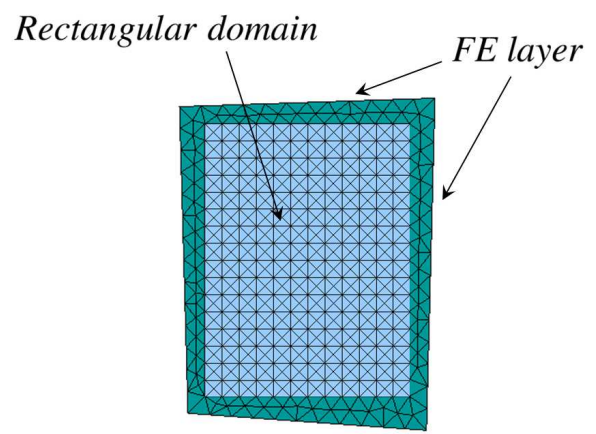

Figure 4: Illustration of a WFE superelement surrounded by a thin FE layer with a non-uniform thickness.

While the rectangular domain exhibits a periodic FE mesh, the layer can be discretized in arbitrary ways. By coupling a WFE-based rectangular element whose dimensions can be large, with a small FE layer, this yields the consideration of a so-called hybrid WFE/FE superelement which is well-suited for model reduction purpose. The modeling of this superelement is achieved as follows.

In matrix form, the dynamic equilibrium equation of the FE layer is expressed as

$$
\left[\begin{array}{ll}
\mathbb{D}_{\mathrm{BB}} & \mathbb{D}_{\mathrm{BR}} \\
\mathbb{D}_{\mathrm{RB}} & \mathbb{D}_{\mathrm{RR}}
\end{array}\right]\left[\begin{array}{c}
\widetilde{\mathbf{p}}_{\mathrm{B}} \\
\widetilde{\mathbf{p}}_{\mathrm{R}}
\end{array}\right]=\left[\begin{array}{c}
-\widetilde{\mathbf{F}}_{\mathrm{B}} \\
\widetilde{\mathbf{F}}_{\mathrm{R}}
\end{array}\right]
$$


where the subscript B refers to the interface DOFs - i.e., where coupling with the WFE-based rectangular superelement occurs - while the subscript $\mathrm{R}$ refers to the remaining DOFs. Also, the tilde sign indicates that vector terms result from the reduced modeling of the WFE-based rectangular superelement, with the assumption that $\widetilde{\mathbf{H}} \widetilde{\mathbf{F}}_{B}=\widetilde{\mathbf{p}}_{B}$ (see after Eq. (23)). Note that the term $-\widetilde{\mathbf{F}}_{\mathrm{B}}$ in the right hand side of Eq. (28) reflects the opposite of the vector of nodal acoustic forces acting on the edges of the rectangular superelement, and results from the action-reaction law. Also, the term $\widetilde{\mathbf{F}}_{\mathrm{R}}$ refers to the vector of nodal acoustic forces acting on the remaining nodes of the FE layer. In left-multiplying the first row block of the matrix system in Eq. (28) by $\mathbb{D}_{\mathrm{BB}} \widetilde{\mathbf{H}}$, where $\widetilde{\mathbf{H}}$ is the receptance matrix of the rectangular domain, this yields

$$
\left[\begin{array}{cc}
\mathbb{D}_{\mathrm{BB}} \widetilde{\mathbf{H}} \mathbb{D}_{\mathrm{BB}} & \mathbb{D}_{\mathrm{BB}} \widetilde{\mathbf{H}} \mathbb{D}_{\mathrm{BR}} \\
\mathbb{D}_{\mathrm{RB}} & \mathbb{D}_{\mathrm{RR}}
\end{array}\right]\left[\begin{array}{c}
\widetilde{\mathbf{p}}_{\mathrm{B}} \\
\widetilde{\mathbf{p}}_{\mathrm{R}}
\end{array}\right]=\left[\begin{array}{c}
-\mathbb{D}_{\mathrm{BB}} \widetilde{\mathbf{H}} \widetilde{\mathbf{F}}_{\mathrm{B}} \\
\widetilde{\mathbf{F}}_{\mathrm{R}}
\end{array}\right]
$$

By using the fact that $\widetilde{\mathbf{H}} \widetilde{\mathbf{F}}_{\mathrm{B}}=\widetilde{\mathbf{p}}_{\mathrm{B}}$, thus it turns out from Eq. (29) that the dynamic equilibrium equation of the hybrid WFE/FE superelement can be expressed as follows:

$$
\left[\begin{array}{cc}
\mathbb{D}_{\mathrm{BB}}+\mathbb{D}_{\mathrm{BB}} \widetilde{\mathbf{H}} \mathbb{D}_{\mathrm{BB}} & \mathbb{D}_{\mathrm{BB}} \widetilde{\mathbf{H}} \mathbb{D}_{\mathrm{BR}} \\
\mathbb{D}_{\mathrm{RB}} & \mathbb{D}_{\mathrm{RR}}
\end{array}\right]\left[\begin{array}{c}
\widetilde{\mathbf{p}}_{\mathrm{B}} \\
\widetilde{\mathbf{p}}_{\mathrm{R}}
\end{array}\right]=\left[\begin{array}{c}
\mathbf{0} \\
\widetilde{\mathbf{F}}_{\mathrm{R}}
\end{array}\right]
$$

\subsubsection{Superelement assembly}

Modeling a whole arbitrary-shaped acoustic cavity involves assembling several hybrid WFE/FE superelements with conventional FE components and CMS superelements, as shown in Figure 5. Within the CMS framework, the condensed dynamic stiffness matrices of superelements may be assessed by considering the Craig-Bampton (CB) method [1], i.e., by means of static modes and fixed interface modes. In matrix form, the dynamic equilibrium equations of these FE components and CB-based superelements may be translated as follows, respectively:

$$
\mathrm{D}^{\mathrm{FE}} \widetilde{\mathbf{p}}=\widetilde{\mathbf{F}}, \quad, \quad \widetilde{\mathrm{D}}^{\mathrm{CB}} \widetilde{\mathbf{p}}_{\mathrm{B}}=\widetilde{\mathbf{F}}_{\mathrm{B}},
$$

where $\mathrm{D}^{\mathrm{FE}}$ is the classic dynamic stiffness matrix of a FE component $(\widetilde{\mathbf{p}}$ and $\widetilde{\mathbf{F}}$ being the vectors of nodal pressures and nodal forces of the component), while $\widetilde{\mathrm{D}}^{\mathrm{CB}}$ is the condensed dynamic stiffness matrix of a superelement, whose expression can be found in [11]; also, the subscript $B$ refers to the boundary DOFs, while the tilde sign of $\widetilde{D}^{\mathrm{CB}}$ indicates that a reduced number of fixed interface modes are used to derive the matrix. On the other hand, the dynamic equilibrium equation of a hybrid WFE/FE superelement is expressed as follows (Eq. (30)):

$$
\widetilde{\mathrm{D}}^{\mathrm{SE}} \widetilde{\mathbf{p}}_{\text {Lay }}=\widetilde{\mathbf{F}}_{\text {Lay }}
$$

where

$$
\widetilde{\mathbf{D}}^{\mathrm{SE}}=\left[\begin{array}{cc}
\mathbb{D}_{\mathrm{BB}}+\mathbb{D}_{\mathrm{BB}} \widetilde{\mathbf{H}} \mathbb{D}_{\mathrm{BB}} & \mathbb{D}_{\mathrm{BB}} \widetilde{\mathbf{H}} \mathbb{D}_{\mathrm{BR}} \\
\mathbb{D}_{\mathrm{RB}} & \mathbb{D}_{\mathrm{RR}}
\end{array}\right]
$$

In Eq. (30), $\widetilde{\mathbf{p}}_{\text {Lay }}$ and $\widetilde{\mathbf{F}}_{\text {Lay }}$ are the vectors of nodal pressures and nodal forces of the layer, expressed by

$$
\widetilde{\mathbf{p}}_{\text {Lay }}=\left[\begin{array}{c}
\widetilde{\mathbf{p}}_{\mathrm{B}} \\
\widetilde{\mathbf{p}}_{\mathrm{R}}
\end{array}\right] \quad, \quad \widetilde{\mathbf{F}}_{\text {Lay }}=\left[\begin{array}{c}
\mathbf{0} \\
\widetilde{\mathbf{F}}_{\mathrm{R}}
\end{array}\right]
$$


The numerical model of a whole cavity composed of several WFE/FE superelements, FE components and $\mathrm{CB}$ superelements hence follows from conventional FE procedure, by assembling several dynamic stiffness matrices $\widetilde{\mathbf{D}}_{r}^{\mathrm{SE}}, \mathbf{D}_{s}^{\mathrm{FE}}$ and $\widetilde{\mathbf{D}}_{t}^{\mathrm{CB}}(r, s, t=1,2, \ldots)$. This yields the following dynamic equilibrium equation

$$
\widetilde{\mathrm{D}}^{\mathrm{Cav}} \widetilde{\mathbf{p}}=\widetilde{\mathbf{F}},
$$

where $\widetilde{\mathbf{p}}$ and $\widetilde{\mathbf{F}}$ are to be understood as the full vectors of nodal pressures and forces of the cavity, respectively. Also, the matrix $\widetilde{\mathbf{D}}^{\mathrm{Cav}}$ is expressed by

$$
\widetilde{\mathbf{D}}^{\mathrm{Cav}}=\sum_{r}\left(\mathbf{L}_{r}^{\mathrm{SE}}\right)^{T} \widetilde{\mathbf{D}}_{r}^{\mathrm{SE}} \mathbf{L}_{r}^{\mathrm{SE}}+\sum_{s}\left(\mathbf{L}_{s}^{\mathrm{FE}}\right)^{T} \mathbf{D}_{s}^{\mathrm{FE}} \mathbf{L}_{s}^{\mathrm{FE}}+\sum_{t}\left(\mathbf{L}_{t}^{\mathrm{CB}}\right)^{T} \widetilde{\mathbf{D}}_{t}^{\mathrm{CB}} \mathbf{L}_{t}^{\mathrm{CB}}
$$

where $\mathbf{L}_{r}^{\mathrm{SE}}, \mathbf{L}_{s}^{\mathrm{FE}}$ and $\mathbf{L}_{t}^{\mathrm{CB}}$ are conventional Boolean localization matrices.

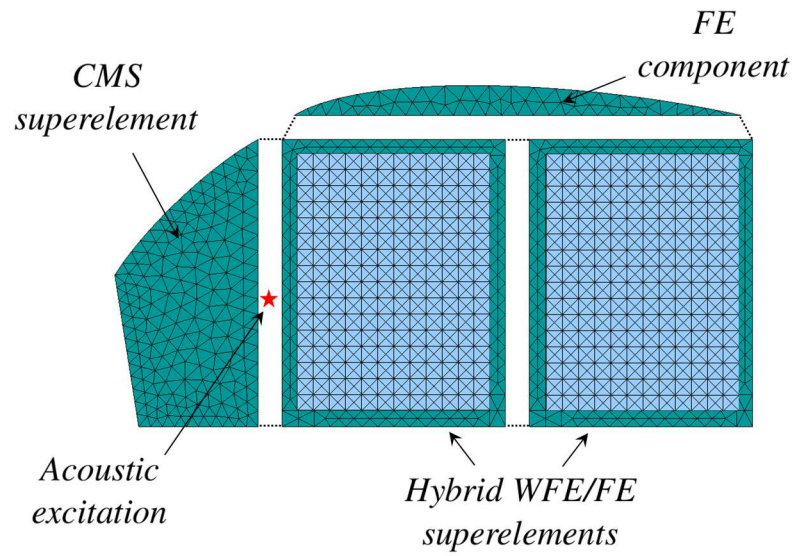

Figure 5: Illustration of an assembly involving two hybrid WFE/FE superelements, one FE component and one CMS superelement.

\section{NUMERICAL RESULTS}

\subsection{Hybrid WFE/FE superelement}

The concept of hybrid WFE/FE superelement is first investigated in the case of a twodimensional rectangular cavity filled with air. Following the strategy depicted in Section 3, this acoustic domain is composed of a WFE-based superelement of dimensions $3 \mathrm{~m} \times 2 \mathrm{~m}$ surrounded by a uniform FE layer of thickness $0.02 \mathrm{~m}$ (Figure 6(a)). Both WFE domain and FE layer are meshed by means of square linear acoustic elements owning 1 DOF per node, of size $0.01 \mathrm{~m} \times 0.01 \mathrm{~m}$ (Figure 6(b)). The FE layer therefore contains 2 elements in its thickness, involving a total number of 3,024 DOFs. The mesh of the WFE-based internal domain consists of $N_{1}=300$ elements in the horizontal $x$-direction and $N_{2}=200$ elements in the vertical $y$-direction. Each left/right and bottom/top edge contains respectively $n_{1}=201$ and $n_{2}=301$ DOFs, the total number of DOFs on the interface between the WFE domain and the FE layer being $n_{\mathrm{B}}=1,000$.

The hybrid WFE/FE superelement modeling is used to investigate the frequency behavior of the cavity which is subject to two acoustic point sources on the external boundary of the FE layer, as shown in Figure 6(a). These acoustic point forces have equal frequency-dependent 
(a)

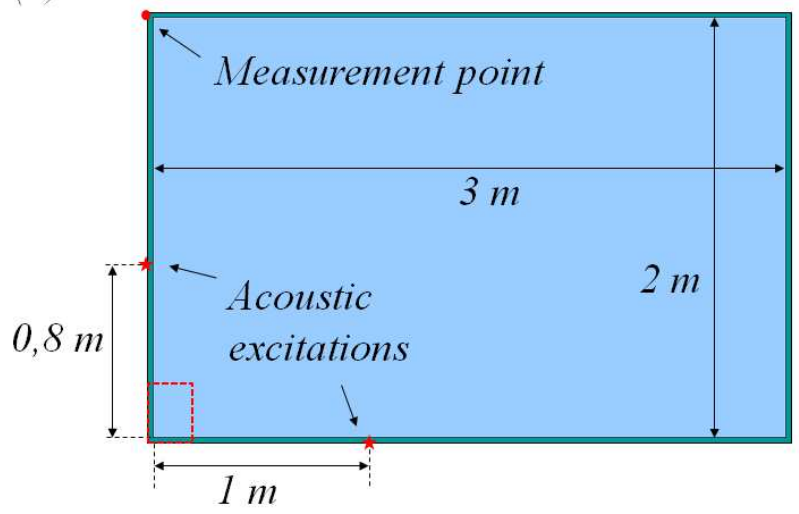

(b)

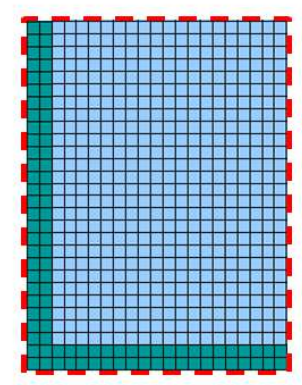

Figure 6: Hybrid WFE/FE superelement: (a) dimensions and test configuration; (b) detailed view of the mesh.

magnitudes of $F_{0}=2 \sqrt{\frac{\omega P}{\rho_{0}}}$ where $P=10^{-6} \mathrm{~W} \cdot \mathrm{m}^{-1}$. The fluid, considered as compressible and barotropic, is characterized by its mean density $\rho_{0}=1.25 \mathrm{~kg} \cdot \mathrm{m}^{-3}$ and sound velocity $c_{0}=343 \mathrm{~m} \cdot \mathrm{s}^{-1}$. Also, a fluid viscosity is taken into account through the Stokes' law of sound attenuation, i.e., the Helmholtz equation that governs the acoustic pressure in the cavity is modified by an attenuation coefficient $\alpha$ :

$$
\Delta p+\left(\frac{\omega}{c_{0}}-i \alpha\right)^{2} p=0 .
$$

The damping is here frequency-dependent and given by $\alpha=\frac{2 \eta \omega^{2}}{3 \rho_{0} c_{0}^{3}}$, where $\eta=18.2710^{-6} \mathrm{~Pa} . \mathrm{s}$ is the dynamic viscosity of air.

The pressure FRF is assessed at the node located at the top left corner of the FE layer (Figure 6(a)), for 246 discrete frequencies uniformly spaced over the range $[10 \mathrm{~Hz}, 500 \mathrm{~Hz}]$ with a frequency step of $2 \mathrm{~Hz}$. The mesh is supposed to be fine enough to accurately capture the dynamic behavior of the system throughout the whole frequency range, i.e., by considering the usual criterion that the acoustic wavelengths should be discretized by a minimum of 8 elements. As was explained in Section 3, the computation of the FRF of the hybrid WFE/FE superelement involves several steps which includes the calculation of matrices of reduced wave bases $\left(\widetilde{\Phi}_{\mathrm{p}}\right)_{i}$, $\left(\widetilde{\boldsymbol{\Phi}}_{\mathrm{p}}^{\star}\right)_{i},\left(\widetilde{\boldsymbol{\Phi}}_{\mathrm{F}}\right)_{i},\left(\widetilde{\boldsymbol{\Phi}}_{\mathrm{F}}^{\star}\right)_{i}$ for each direction $i=1,2$. These are used to form the $n_{\mathrm{B}} \times n_{\mathrm{B}}$ receptance matrix $\widetilde{\mathbf{H}}$, see Eq. (25). The receptance matrix is finally combined to the dynamic stiffness matrix $\mathbb{D}$ of the FE layer to form the matrix $\widetilde{\mathrm{D}}^{\mathrm{SE}}$ (Eqs. (30), (33)) of the hybrid WFE/FE superelement, with a view to solving the matrix system (35) at each frequency of interest.

The motivation behind the use of the FE layer, around the WFE domain, is to highly reduce the sizes of the wave bases without significantly altering the accuracy of the solution. In the present case, the pressure FRF has been computed by only retaining $m_{1}=20$ right/left- and $m_{2}=20$ top/bottom-going wave modes among the $n_{1}=201$ and $n_{2}=301$ modes of the full wave bases. The resulting dB pressure levels, i.e., $20 \log _{10}\left(\frac{|p|}{p_{\text {ref }}}\right)$ where $p_{\text {ref }}=20 \times 10^{-6} P a$, are shown in Figure 7 (dotted pink line). For validation purpose, a reference FE solution issued from the commercial FE software Comsol Multiphysics ${ }^{\circledR}$ is also displayed in Figure 7 (black solid line). It can be seen that the reference levels are correctly retrieved by the hybrid superelement approach over the whole frequency band, despite a few slight differences regarding some lowfrequency anti-resonance peaks and the resonance peak at $f=378 \mathrm{~Hz}$. 
(a)

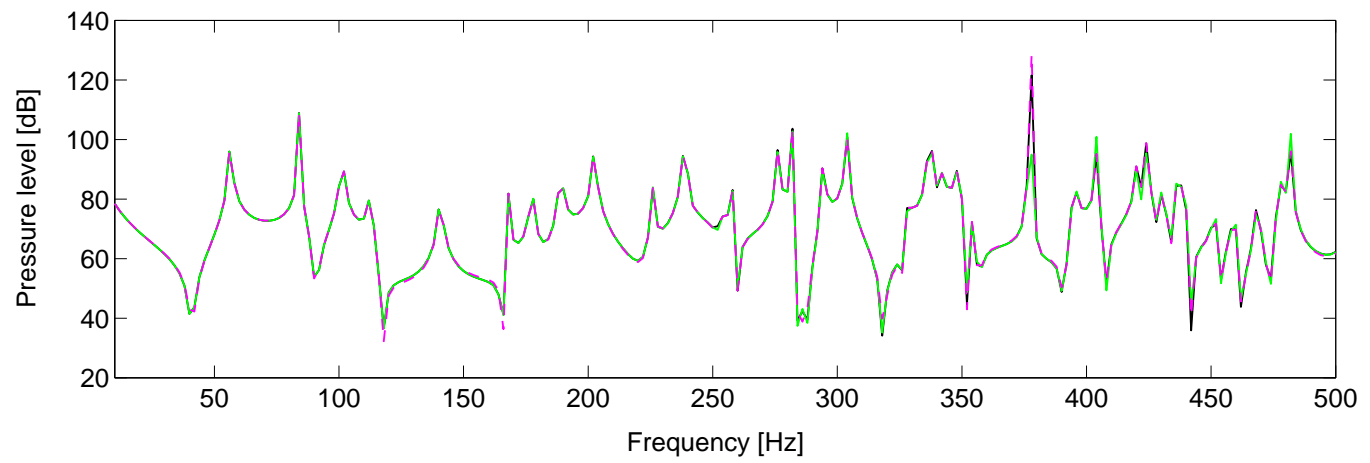

(b)

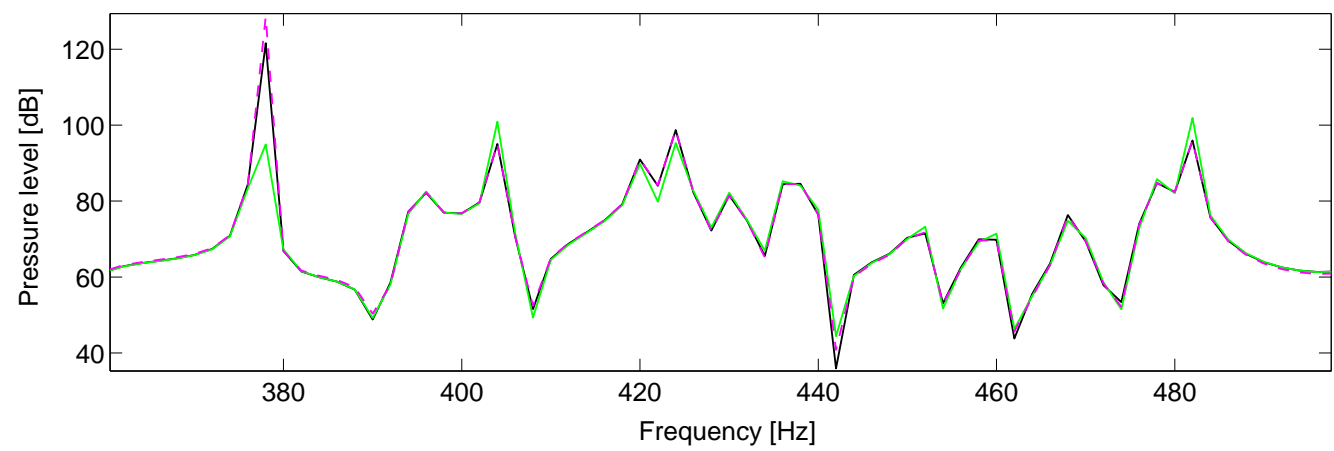

Figure 7: Pressure FRFs of the rectangular hybrid WFE/FE cavity: (-) FE reference solution; (- -) WFE/FE solution; (-) CB solution with 1,000 elastic modes; (a) full frequency range; (b) focus in the range [360 Hz, $500 \mathrm{~Hz}]$.

Regarding CPU times, it takes as a whole $852 \mathrm{~s}$ to achieve the computation of the pressure FRF (including the computations of wave modes) with MATLAB ${ }^{\circledR}$ and using an Intel ${ }^{\circledR}$ Core(TM) i7-3720QM @2.6 GHz processor. By considering full wave bases instead of reduced wave bases, it would have taken 1,888 s to compute the FRF. Thus the use of reduced wave bases yields a reduction of $54.9 \%$ of the computational cost, hence giving credit to the proposed model reduction strategy.

Also, the efficiency of the method is assessed when compared to the Craig-Bampton (CB) method [1]. In this framework, the condensed dynamic stiffness matrix $\widetilde{D}^{C B}$ of the cavity is modeled by means of 1,016 static modes and 1,000 fixed interface modes. The related FRF is displayed in Figure 7 in green solid line. As it can be seen in Figure 7(b), the CB solution fails to accurately describe the resonance peaks of the reference FE solution above $300 \mathrm{~Hz}$, as opposed to the hybrid WFE/FE-based approach. Besides, the CPU time involved in the CB approach is $966 \mathrm{~s}$, which exceeds that of the hybrid WFE/FE-based approach. To summarize, it appears from the analysis of this first test case that the hybrid WFE/FE-based approach constitutes an efficient alternative to the $\mathrm{CB}$ method.

\subsection{Superelement assembly}

In the following, the above rectangular cavity is embedded in an arbitrary-shaped assembly depicted in Figure 8. The latter is composed of a hybrid WFE/FE superelement which is connected to a CMS superelement, modeled by means of the CB method. The system is excited by means of an acoustic point source located inside the CMS superelement. In the present case, 
the hybrid WFE/FE superelement is made up of a WFE-based rectangular domain which is connected to a non-uniform FE layer over two of its edges only. In doing so, large reduction of the sizes of the WFE wave bases is expected over a reasonable frequency range - which is chosen to be $[10 \mathrm{~Hz}, 300 \mathrm{~Hz}]$ — without significantly penalizing the accuracy of the proposed approach.

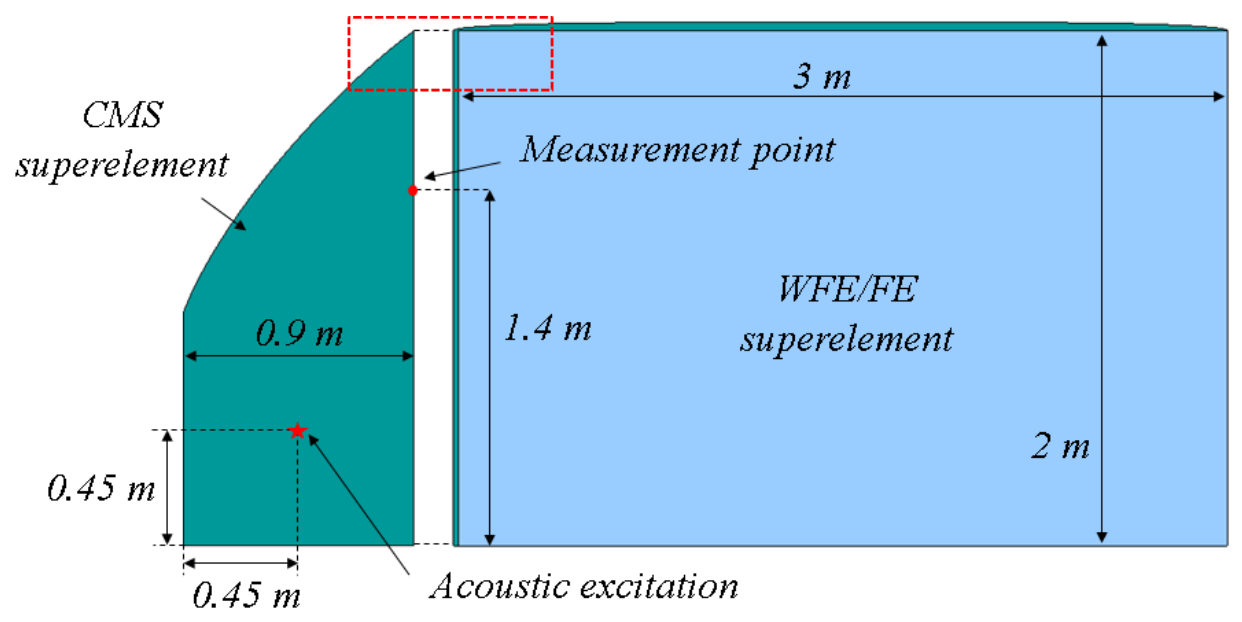

Figure 8: Assembly of a hybrid WFE/FE superelement and a CMS superelement: dimensions and test configuration.

The WFE-based rectangular domain is meshed as in the previous case, i.e., using square linear elements of size $0.01 \mathrm{~m} \times 0.01 \mathrm{~m}$. Regarding the FE layer, square linear elements are used in the vertical portion of the layer, as well as triangular linear elements in the curved portion at the top of the WFE domain (Figure 9). The mesh of the FE layer hence contains 1,698 DOFs, including 501 DOFs on the interface with the WFE domain. Finally, the mesh of the CMS superelement is composed of triangular and rectangular linear elements, which involves a total of 12,047 DOFs including 530 DOFs uniformly spread on the superelement boundary. Within the framework of the present approach, two dynamic stiffness matrices $\widetilde{\mathrm{D}}^{\mathrm{SE}}$ and $\widetilde{\mathrm{D}}^{\mathrm{CB}}$ are thus involved which are of respective sizes $875 \times 875$ and $530 \times 530$, while the full assembly contains 73,540 DOFs.

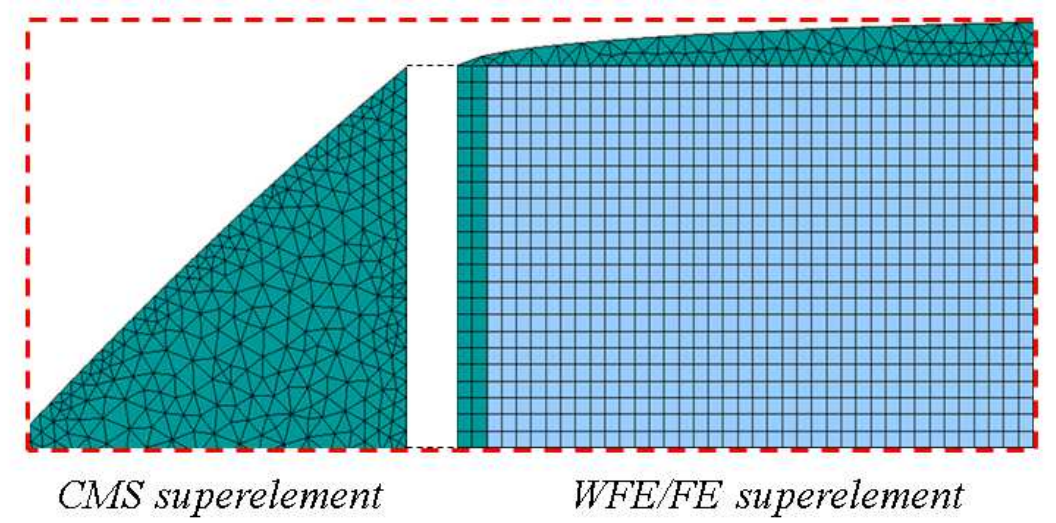

Figure 9: Detailed view of the assembly meshes.

The pressure FRF is computed at a node located on the interface between the CMS and 
hybrid WFE/FE superlements, as shown in Figure 8. The resulting pressure levels are plotted in Figure 10. Again, $m_{1}=20$ right/left- and $m_{2}=20$ top/bottom-going wave modes are retained for modeling the hybrid WFE/FE superelement, while 500 fixed interface modes are considered for modeling the CMS/CB superelement. Again, the WFE-based solution correctly matches the FE reference solution over the whole frequency range, despite some very slight differences above $200 \mathrm{~Hz}$ which appear to be less than $3 \mathrm{~dB}$, however.
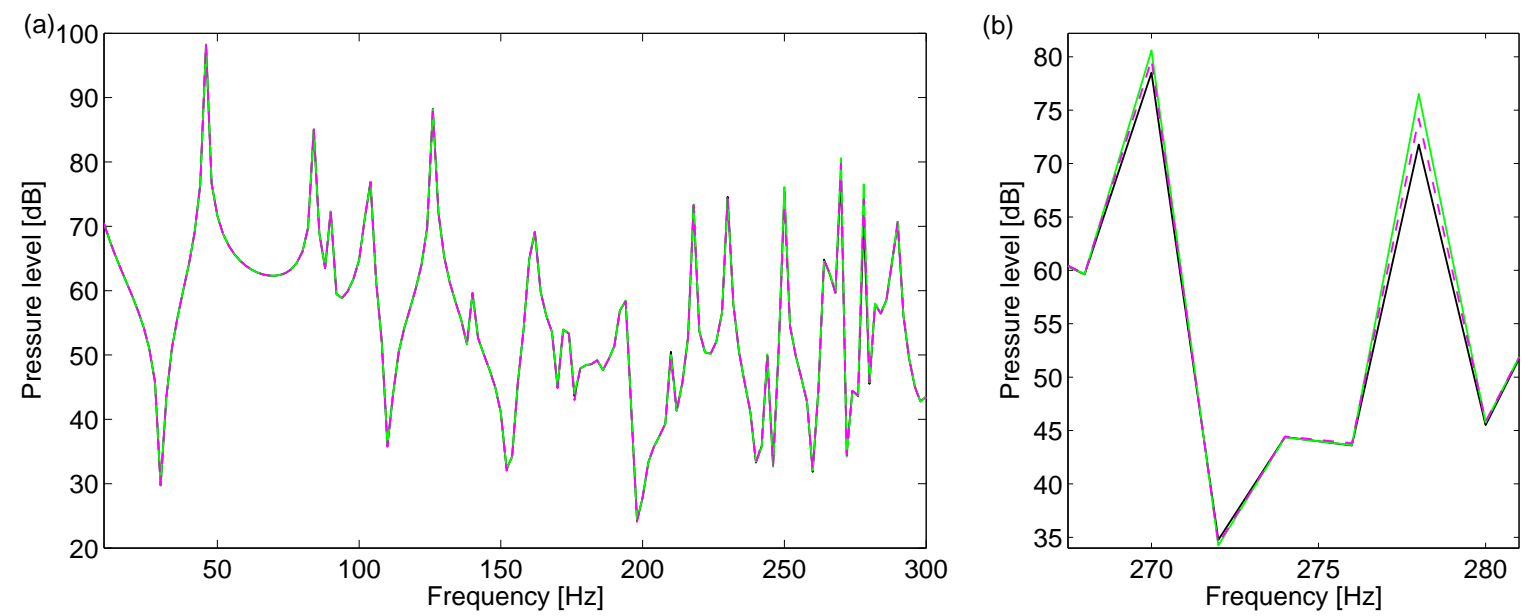

Figure 10: Pressure FRFs of the assembly: (-) FE reference solution; (- - ) solution obtained with the hybrid WFE/FE superelement and a CB superelement with 500 fixed interface modes; (-) solution obtained using two CB superelements with 500 and 1000 fixed interface modes; (a) full frequency range; (b) focus in the range [267 Hz, $281 \mathrm{~Hz}]$.

The efficiency of the method is assessed in comparison with the conventional CB method. For this purpose, two CMS/CB superelements are considered which concern the one depicted in Figure 8 and the previous WFE/FE superelement. Here, 500 and 1, 000 fixed interface modes are respectively used for modeling these superelements. The CB-based pressure FRF is displayed in Figure 10, which appears to be a little bit less accurate than the WFE-based solution, as shown Figure 10(b). The interesting feature of the proposed hybrid WFE/FE modeling lies in the reduction of the CPU times. It actually takes $433 \mathrm{~s}$ for computing the pressure FRF with the hybrid WFE/FE-based approach, compared to $905 \mathrm{~s}$ with the conventional CB method. This means $52.2 \%$ of time reduction, which fully gives credit to the proposed approach.

\section{CONCLUSIONS}

A new substructuring technique has been proposed for the prediction of the acoustic behavior of arbitrary-shaped 2D cavities. It involves WFE/FE hybrid superelements, consisting in a rectangular domain, with a periodic FE mesh and modeled by means of the WFE method, surrounded by a FE layer with a small width. The interesting feature behind this modeling is that a few wave modes are only required to describe the dynamic stiffness matrices of those WFE/FE hybrid superelements, meaning that they can be computed in a very fast way. Modeling a whole arbitrary-shaped acoustic cavity follows from conventional FE assembly procedure, i.e., by coupling several WFE/FE hybrid superelements together as well as with other CMS superelements and classical FE components. Numerical experiments have been carried out which highlight the relevance of the proposed substructuring technique, in terms of accuracy and computational 
saving, in comparison with the conventional CMS technique.

\section{REFERENCES}

[1] R. R. Craig, M. C. C. Bampton, Coupling of substructures for dynamic analyses, AIAA Journal 6 (7) (1968) 1313-1319.

[2] B. V. Genechten, B. Pluymers, D. Vandepitte, W. Desmet, A hybrid wave based - modally reduced finite element method for the efficient analysis of low- and mid-frequency car cavity acoustics, SAE International Journal of Passenger cars - Mechanical systems 2 (1) (2009) 1494-1504.

[3] W. X. Zhong, F. W. Williams, On the direct solution of wave propagation for repetitive structures, Journal of Sound and Vibration 181 (3) (1995) 485-501.

[4] J.-M. Mencik, M. N. Ichchou, Multi-mode propagation and diffusion in structures through finite elements, European Journal of Mechanics - A/Solids 24 (5) (2005) 877-898.

[5] D. Duhamel, B. Mace, M. J. Brennan, Finite element analysis of the vibrations of waveguides and periodic structures, Journal of Sound and Vibration 294 (1-2) (2006) 205-220.

[6] R. Patel, On computing the eigenvalues of a symplectic pencil, Linear Algebra and its Applications 188-189 (1993) 591-611.

[7] J.-M. Mencik, D. Duhamel, A wave-based model reduction technique for the description of the dynamic behavior of periodic structures involving arbitrary-shaped substructures and large-sized finite element models, Finite Elements in Analysis and Design (accepted).

[8] R. S. Langley, Wave evolution, reflection, and transmission along inhomogeneous waveguides, Journal of Sound and Vibration 227 (1) (1999) 131-158.

[9] J.-M. Mencik, On the low- and mid-frequency forced response of elastic systems using wave finite elements with one-dimensional propagation, Computers and Structures 88 (1112) (2010) 674-689.

[10] J.-M. Mencik, New advances in the forced response computation of periodic structures using the wave finite element (WFE) method, Computational Mechanics 54 (3) (2014) 789-801.

[11] J.-M. Mencik, Model reduction and perturbation analysis of wave finite element formulations for computing the forced response of coupled elastic systems involving junctions with uncertain eigenfrequencies, Computer Methods in Applied Mechanics and Engineering 200 (45-46) (2011) 3051-3065. 